\title{
An Iterative Model for the Steady-State Current Distribution in Oxide-Confined VCSELs
}

\author{
Hsueh-hua Chuang, Student Member, IEEE, James R. Biard, Life Fellow, IEEE, Jim Guenter, Senior Member, IEEE, \\ Ralph Johnson, Gary A. Evans, Fellow, IEEE, and Jerome K. Butler, Fellow, IEEE
}

\begin{abstract}
This paper presents an iterative model for the analysis of the current distribution in vertical-cavity surface-emitting lasers (VCSELs) using a SPICE-like approach. The model includes a degeneracy correction for operation at and above threshold. The effect of the resistance due to the p-distributed Bragg reflector (p-DBR) mirror layers and the oxide layer on performance is investigated. Higher sheet resistance under the oxide layer reduces the threshold current, but reduces the current range over which single transverse mode operation occurs. The voltage drop across the p-DBR region dominates spatial hole burning, which is moderated by lateral drift and diffusion. This simple iterative model is applied to commercially available oxide-confined VCSELs.
\end{abstract}

Index Terms-Drift and diffusion, semiconductor lasers, spatial hole burning (SHB), surface-emitting laser.

\section{INTRODUCTION}

$\mathbf{V}$ ERTICAL-CAVITY surface-emitting lasers (VCSELs) provide low threshold current, a symmetrical optical beam, single-longitudinal-mode operation, and wafer-level testing. Applications include telecommunications, optical interconnects, and optical mice and displays. VCSELs also allow monolithic integration of additional photonic and electronic components. Despite the above advantages, their complexity makes performance optimization a challenge. For example, spatial hole burning (SHB) of the transverse-mode profile can cause nonuniformities in the local carrier concentration in the quantum wells, resulting in a secondary pulse in the turn-off transient or a slow turn-off time [1].

The carrier and current distributions in VCSELs involve multiple processes, including carrier transport and recombination. Extensive analysis of the physical phenomena in VCSELs [2]-[6] includes 2-D current flow, lateral thermal distributions, and optimization of heterostructure distributed Bragg reflectors (DBRs). Hadley et al. [2] developed a 2-D model including heat transfer and accounted for multiple lateral modes. Bissessur et al. [3] later developed a quasi-3D model including carrier transport using a beam-propagation analysis

Manuscript received February 23, 2007; revised June 28, 2007. This work was supported in part by the Texas Higher Education Coordinating Board under Grant 003613-0041-2003, in part by a Gerald J. Ford Research Fellowship, and in part by an Air Force Office of Scientific Research STTR Contract FA9550-06-C-0146.

H. Chuang, G. A. Evans, and J. K. Butler are with the Electrical Engineering Department, Southern Methodist University, Dallas, TX 75275-0338 USA (e-mail: jhhchuang@gmail.com; hchuang@engr.smu.edu; gae@engr.smu.edu; jkb@engr.smu.edu).

J. R. Biard, J. Guenter, and R. Johnson are with Finisar, Allen, TX 75013 USA (e-mail: bob.biard@ finisar.com; Jim.Guenter@ finisar.com; Ralph.Johnson@finisar.com).

Digital Object Identifier 10.1109/JQE.2007.905005 that included scattering losses. More recently, Streiff et al. [4] implemented a detailed electrical-thermal-optical simulator by using a finite-element method for a mesa VCSEL. Their results showed good agreement with measured light-current $(L-I)$ and current-voltage $(I-V)$ curves. Osiński [5] also showed a self-consistent electrical-thermal-optical model for an oxide VCSEL and addressed current distribution variations with applied voltage. A resistance network was used to solve the current distribution in VCSELs using SPICE [6].

In [2] and [7], ambipolar diffusion was included in the carrier diffusion calculation. However, in our iterative model, drift diffusion is used to avoid the ambiguities of the ambipolar approach.

In this paper, we use a simple SPICE-like model that includes both resistors and diodes to show the impact of SHB due to the high series resistance and the anisotropy of the resistivity of the p-DBR mirror on the current distribution within the VCSEL. Most simple theoretical models are based on a rate-equation approach. To our knowledge, all rate-equation approaches assume either a uniform current density or a uniform potential. This constraint is a major problem for VCSELs, because the resistivity of the DBR layers is highly anisotropic. As a consequence, the rate-equation approach to VCSELs has difficulty handling series resistance effects without using finite elements.

We want to know the details of the current density on a microscopic level compared with the scale of the optical mode. Therefore, we consider numerous annular segments of the VCSEL geometry, within which the current density is assumed constant (and therefore the rate equation is satisfied in each annular element).

This purpose of this paper is not to provide a complete analysis of VCSELs, but to determine how layer thicknesses, compositions, and dopings, particularly in the p-DBR mirror region, effect the current distribution as a function of bias current. The iterative model developed in this paper shows that hole burning due to anisoptropy of the resisitivity of the p-DBR mirror contributes to mode changes. This model can also be used to explore design changes to increase the onset of higher order modes to higher power levels.

Process monitors are used during VCSEL fabrication to measure the sheet resistances of the p-DBR mirror and the region under the oxide layer along with the nkT surface recombination current [8]. These measured values, shown in Table I for a particular VCSEL structure, are used in our SPICE-like model to find the current distribution within the VCSEL as a function of bias current. Further, by introducing adjustable parameters in our SPICE-like model that relate to the resistivity anisotropy of the p-DBR mirror, we can fit the predicted light—current $(L-I)$ 
TABLE I

MEASURED PARAMETERS OBTAINED From Process MONITORS USED IN THE ITERATIVE MODEL

\begin{tabular}{llcc}
\hline \hline Symbol & \multicolumn{1}{c}{ Parameters } & Units & Value \\
\cline { 2 - 4 } & aperture radius & $\mu \mathrm{m}$ & 5.0 \\
$R_{\mathrm{spdbr}}$ & p-DBR mirror sheet resistance & $\Omega / \square$ & 43.5 \\
$R_{\mathrm{so}}$ & sheet resistance under the oxide layer & $\Omega / \square$ & 3165 \\
$n$ & ideality factor for surface & - & 1.907 \\
& recombination current & & \\
\hline \hline
\end{tabular}

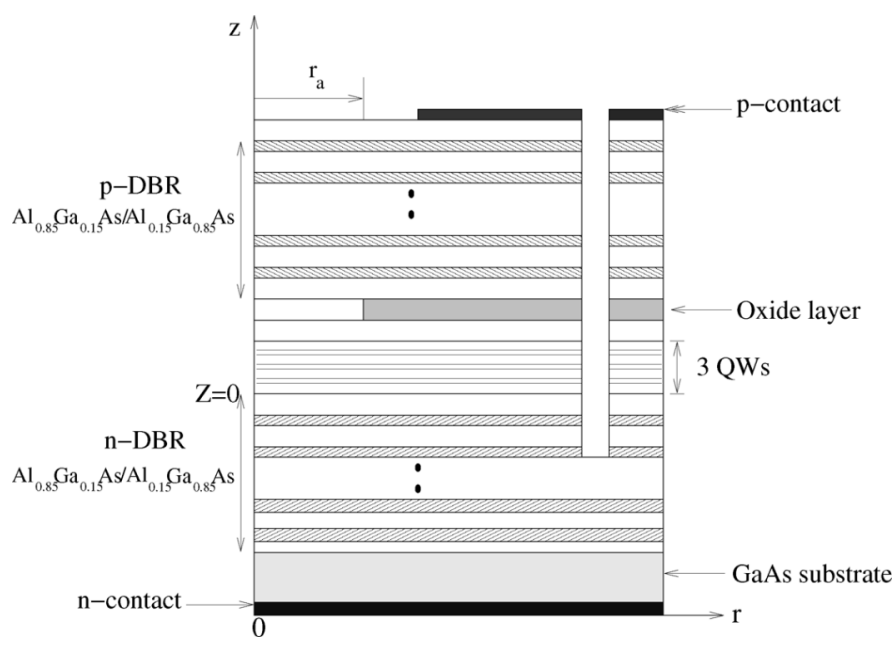

Fig. 1. Sketch of the right-hand side of a cylindrically symmetric oxide confined VCSEL with three $\mathrm{GaAs} / \mathrm{Al}_{0.25} \mathrm{Ga}_{0.75} \mathrm{As} \mathrm{QWs}$. The p-contact forms a contact ring.

and current-voltage $(I-V)$ curves to the experimentally measured curves. With an optimum fit, we can obtain the microscopic current distributions as a function of drive current within a VCSEL.

In Section II, we introduce a circuit grid and derive the relevant equations for solving the voltages in the VCSEL. Different lateral current calculation schemes and simulations with and without bandgap renormalization are performed to show the effects on the VCSEL performance. Section III investigates SHB. Finally, in Section IV, simulation results are compared with experimental data.

\section{DESCRIPTION OF THE MODEL}

We present an iterative model for the analysis of the current distribution in a cylindrical 850-nm VCSEL and use a SPICElike approach to solve the equations. Fig. 1 shows the right-hand side of a circularly symmetric VCSEL with a ring contact on the top (p-side). The current-confining aperture is fabricated by a wet thermal oxidation process that converts $\mathrm{Al}_{\mathrm{x}} \mathrm{Ga}_{1-\mathrm{x}}$ As to $\mathrm{Al}_{\mathrm{x}} \mathrm{O}_{\mathrm{y}}$. The opening of the oxide layer defines the cavity radius. The active region consists of three $70-\AA$ GaAs quantum wells (QWs) separated by $100-\AA$-thick $\mathrm{Al}_{0.25} \mathrm{Ga}_{0.75}$ As barriers. The isolation trench extends to the n-DBR region.

The circuit network shown in Fig. 2 represents the VCSEL structure shown in Fig. 1. The resistors in Fig. 2 represent the resistance from the p-DBR layers and the layers under the oxide layer. The diodes represent the three-QW active region. The top

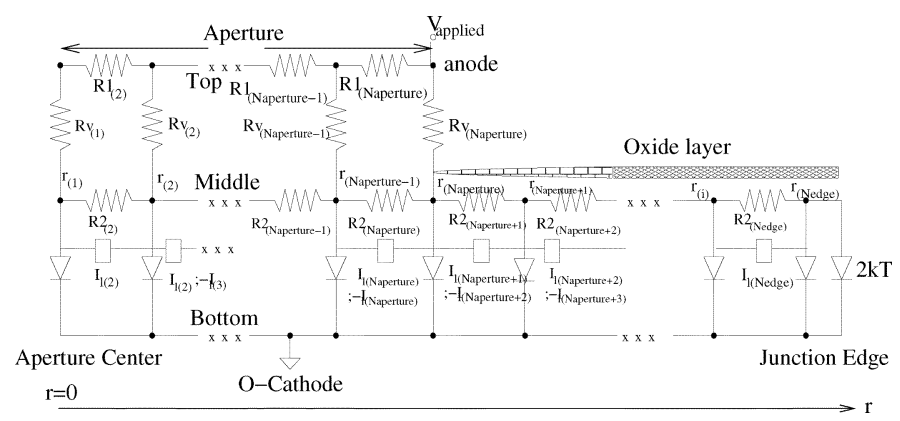

Fig. 2. Circuit diagram used for the iterative model of the VCSEL in Fig. 1. The nodes below the diodes are equal-potential. Each vertical resistor or diode is contained within an annular region. Horizontal resistors connect annular regions.

of the n-DBR is assumed to be an equal-potential surface because of the higher mobility of electrons and the large surface area of the n-contact.

Here, we describe how the values of each resistor in Fig. 2 are obtained. The radiative recombination current is the sum of the spontaneous and stimulated currents in the active region. A degeneracy correction for the spontaneous current is necessary above threshold [9]. The $2 \mathrm{kT}$ surface recombination current [8] at the edge of the device is included in the model as shown in Fig. 2. The lateral currents (due to drift and diffusion) flowing in the QWs are represented by the diode connections with boxes containing $I_{1(i)}$ in Fig. 2. The algorithms for finding the voltages and currents in the network shown in Fig. 2 and the models for bandgap renormalization are discussed below and in the Appendix.

\section{A. Resistance}

The circuit in Fig. 2 corresponds to the VCSEL geometry from the top of the p-DBR mirror to the top of the n-DBR mirror. All the resistors are related to the sheet resistance $\left(R_{\mathrm{spdbr}}\right)$ of the p-DBR region. The resistors only under the oxide layer are related to the sheet resistance $\left(R_{\mathrm{so}}\right)$ under the oxide layer. Each diode defines an annular element in the VCSEL geometry, except that, in the center of the cavity $(r=0)$, the element has a circular shape.

The p-DBR mirror consists of 22 pairs of alternating quarter wave thicknesses of $\mathrm{Al}_{0.85} \mathrm{Ga}_{0.15} \mathrm{As}$ and $\mathrm{Al}_{0.15} \mathrm{Ga}_{0.85} \mathrm{As}$. Conduction in the p-DBR mirror is highly asymmetric with the lateral conduction being much higher than the vertical conduction. This is due to a combination of the large variation of mobilities in the vertical direction, vertical variations in doping, and depletion and accumulation effects in and around the graded transition between the layers due to the different electron affinity values of the alternating layers. All of these effects contribute to the overall voltage drop in the DBR [10].

In addition to the resistance of the p-DBR mirror, the resistance under the oxide layer is obtained from the current flowing in the layers under the oxide layer. The resistor network shown in Fig. 2 includes three types of resistors: resistors in row one, resistors in row two, and vertical resistors connecting rows one and two. The lateral resistance of the p-DBR region is distributed between the resistors in row one and row two. The adjustable parameter $\gamma_{21}$ is the ratio of the resistances of the 
resistors in row two to the resistances of the resistors in row one. The values of the resistors in both rows are related to the vertical doping profile in the p-DBR mirror.

The resistance of the $i$ th resistor in row two $\left(R_{2(i)}\right)$ is related to the measured sheet resistance of the p-DBR $\left(R_{\text {spdbr }}\right)$ and the measured sheet resistance under the oxide layer $\left(R_{\text {so }}\right)$ by

$$
\begin{aligned}
& R_{2(i)}=R_{\text {spdbr }} S_{(i)}\left(1+\gamma_{21}\right), \quad 1 \leq i \leq N_{\text {aperture }} \\
& R_{2(i)}=R_{\text {so }} S_{(i)}, \quad N_{\text {aperture }}<i \leq N_{\text {edge }}
\end{aligned}
$$

The total number of squares $S_{(i)}$ in an annular element is

$$
S_{(i)}=\int_{\mathrm{r}_{(i-1)}}^{r_{(i)}} \frac{d r}{2 \pi r}, \quad 1<i \leq N_{\text {edge }}
$$

so

$$
\begin{aligned}
S_{(i)} & =\frac{1}{4 \pi}, \quad i=1 \\
S_{(i)} & =\frac{1}{2 \pi} \ln \left(\frac{r_{(i)}}{r_{(i-1)}}\right), \quad 1<i \leq N_{\text {edge }}
\end{aligned}
$$

where $r_{(i)}$ is the radius of the $i$ th annular element. The lateral resistance of the $i$ th element inside the aperture consists of the resistors in row one in parallel with the resistors in row two. The value of the resistors in row one $R_{1(i)}$ is

$$
R_{1(i)}=\frac{R_{2(i)}}{\gamma_{21}}, \quad 1 \leq i \leq N_{\text {aperture }}
$$

The resistance of the vertical resistors $R_{v}$ can be found from

$$
R_{v(i)}=\frac{\rho_{\mathrm{l}} \gamma_{\mathrm{vl}} t_{p}}{A_{(i)}}, \quad 1 \leq i \leq N_{\text {aperture }}
$$

where $t_{p}$ is the thickness of the p-DBR mirror, $A_{(i)}$ is the area of each element, and $\gamma_{\mathrm{vl}}$, which is another adjustable parameter, is the anisotropy ratio between the vertical and lateral resistivity. The lateral resistivity is $\rho_{1}=R_{\text {spdbr }} t_{p}$. The product of $\rho_{1}$ and $\gamma_{\mathrm{vl}}$ is the vertical resistivity.

The values of $\gamma_{\mathrm{vl}}$ and $\gamma_{21}$ are adjusted to obtain an optimum match of the $L-I$ and $I-V$ curves. This analysis provides valuable information about the anisotropy of the p-DBR mirror that cannot be found any other way.

\section{B. Spontaneous Current With Degeneracy Correction}

In the QWs, direct electron-hole recombination is a radiative recombination process. From Fermi's Golden rule, the spontaneous emission rate $R_{\mathrm{sp}}$ is [11]

$$
R_{\mathrm{sp}}(n)=B n p
$$

where $B$ is the bimolecular radiative recombination constant and $n$ and $p$ are the electron and hole concentrations, respectively. Under nondegenerate conditions, the carrier concentrations in the active region satisfies

$$
n p=n_{i}^{2} \exp \left(\frac{q V}{k T}\right)
$$

where $n_{i}$ is the intrinsic carrier concentration, $q$ is the electron charge, and $V$ is the forward voltage of the VCSEL pn junction.
In each annular element, the carrier-density rate equation for electrons in the QWs is [12]

$$
\frac{\partial n}{\partial t}=D_{\mathrm{n}}\left(\nabla^{2} n\right)+\frac{J}{q d}-R_{\mathrm{sp}}(n)
$$

where $D_{\mathrm{n}}$ is the electron diffusion coefficient, $d$ is the active region thickness (approximated by the total thickness of the quantum wells), and $J$ is the current density. The first term in (8) accounts for diffusion and can be ignored because of the thin active region in the $z$-direction and the constant carrier concentration inside each element. Assuming charge neutrality, $n=p$ and $J=q B d n^{2}$. Thus, the spontaneous current density for the non-degenerate case $\left(J_{\mathrm{sp}, \mathrm{ND}}\right)$ is

$$
J_{\mathrm{sp}, \mathrm{ND}}=q d B n_{i}^{2} \exp \left(\frac{V}{k T}\right) .
$$

The carrier concentration in (7) assumes the quasi-Fermi levels for both electrons and holes are a few $\mathrm{kT}$ below the conduction band edge (the "nondegenerate" case) or above the valence band edge. With increased current injection, the separation of the quasi-Fermi levels increases and becomes equal to or larger than the band gap. In this manner, a population inversion is achieved, providing optical gain. In this case, a degeneracy correction for (9) is necessary.

We assume the current density for the degenerate case $\left(J_{\mathrm{D}}\right)$ has a similar exponential dependence as the nondegenerate current $\left(J_{\mathrm{ND}}\right)$ and that we can write the ratio of the degenerate current density to the nondegenerate current density as

$$
\frac{J_{\mathrm{D}}}{J_{\mathrm{ND}}}=\exp \left(\frac{\Delta V}{k T}\right)
$$

where $\Delta V=V_{\mathrm{D}}-V_{\mathrm{ND}}$ and $V_{\mathrm{D}}$ is the applied voltage in the degenerate case. The nondegenerate forward voltage $\left(V_{\mathrm{ND}}\right)$ is found from (9) by choosing an initial point that is not degenerate (say $V_{\mathrm{f}}=V_{1}=1.16 \mathrm{~V}$ in Fig. 3) with a corresponding current density $J_{1}$ as

$$
V_{\mathrm{ND}}=k T \ln \left(\frac{J}{J_{1}}\right)+V_{1} \text {. }
$$

The quasi-Fermi level separation $V_{\mathrm{f}}$ that is equal to the degenerate forward voltage can be found from the quasi-Fermi-level positions for the electrons $F_{\mathrm{c}}$ and the holes $F_{v}$. The carrier concentration for electrons is given by [13]

$$
n=\sum_{j=1}^{j^{\prime}} N \ln \left\{1+\exp \left[\frac{\left(F_{\mathrm{c}}-E_{j}\right)}{k T}\right]\right\}
$$

where $N=\overline{m_{\mathrm{e}}^{*}} k T / \pi \hbar^{2} \mathrm{~d}, \hbar$ is the Planck's constant divided by $2 \pi, \overline{m_{\mathrm{e}}^{*}}$ is the average electron effective mass from the well and the barrier, and $E_{j}$ is the $j$ th energy level of the subband. The quasi-Fermi level for the electrons can be found from (12). Similarly, the quasi-Fermi level for the holes can be determined from

$$
p=\sum_{j=1, \mathrm{~h}(=l h, h h)}^{j^{\prime}} P \ln \left\{1+\exp \left[\left(\frac{\left.E_{j, \mathrm{~h}}-F_{v}\right)}{k T}\right]\right\}\right.
$$




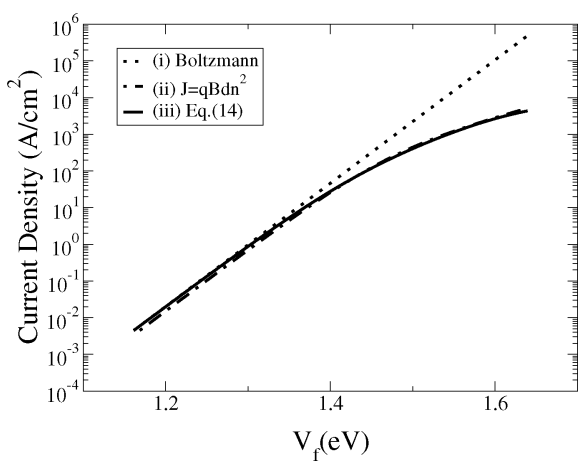

Fig. 3. Current density as a function of quasi-Fermi-level separation. The dotted line (i) is the Boltzmann approximation (nondegenerate case). The dashed line (ii) uses (12) and (13) for the electron and hole quasi-Fermi levels, and $J=q B d n^{2}$. The solid line (iii) includes the degeneracy correction for the Boltzmann approximation.

where $P=\overline{m_{\mathrm{h}}^{*}} k T / \pi \hbar^{2} \mathrm{~d}, \overline{m_{\mathrm{h}}^{*}}$ is the average hole effective mass for the light hole or heavy hole, and $E_{j, \mathrm{~h}}$ is the $j$ th energy level for the heavy-hole or light-hole subband. Using $V_{\mathrm{f}}$ in (10) for the degeneracy correction, the spontaneous current density becomes

$$
J_{\mathrm{sp}}=J_{1} D_{\mathrm{F}}\left(V_{\mathrm{f}}\right) \exp \left(\frac{V_{\mathrm{f}}}{k T}\right)
$$

where

$$
D_{\mathrm{F}}\left(V_{\mathrm{f}}\right)=\frac{1}{\left[1+\phi_{1} \exp \left(\frac{V_{\mathrm{f}}}{m_{\mathrm{D}} k T}\right)+\phi_{2} \exp \left(\frac{V_{\mathrm{f}}}{k T}\right)\right]}
$$

is the degeneracy correction factor, $\phi_{1}, \phi_{2}$, and $m_{\mathrm{D}}$ are the fitting parameters with the value of $3.734 \times 10^{-12}, 1.6 \times 10^{-26}$, and 2.09, respectively. Fig. 3 shows the current density as a function of quasi-Fermi-level separation calculated by three methods: 1) from (9) (Boltzmann); 2) from $J=q B d n^{2}$ combined with (12) and (13); and 3) from (14). Methods 2) and 3) provide results that agree within 3\%. However, under low Fermi-level separation (the nondegenerate condition), all methods provide accurate results.

The spontaneous current for each diode can be described with a degeneracy correction factor as a function of the voltage of each middle node $V_{m(i)}$ in Fig. 2 as

$$
I_{\mathrm{sp}(i)}=A_{(i)} J_{1} \exp \left(\frac{V_{m(i)}}{k T}\right) D_{\mathrm{F}}\left(V_{m(i)}\right) \text {. }
$$

\section{Surface Recombination}

The additional diode at the junction edge (right-hand side of Fig. 2) accounts for surface recombination current. This surface recombination rate is [14]

$$
R(n)=S n
$$

where $S$ is the surface recombination velocity. Similar to the analysis of $k T$ current above, we can obtain a $2 k T$ surface recombination current per unit length given by

$$
I_{\mathrm{s}}=q d S n_{i} \exp \left(\frac{V}{2 k T}\right)
$$

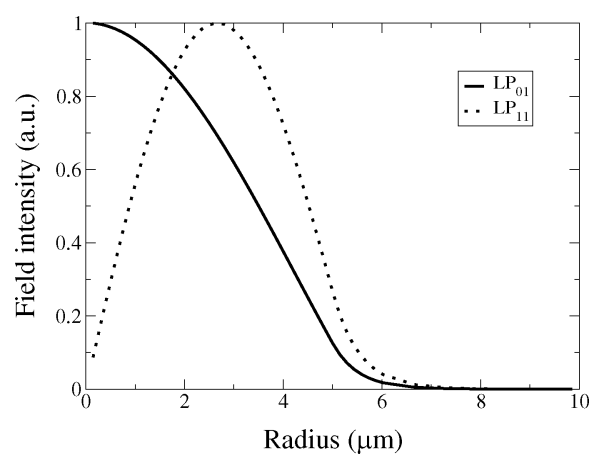

Fig. 4. Optical intensity for the fundamental $\left(\mathrm{LP}_{01}\right)$ mode and the first-order $\left(\mathrm{LP}_{11}\right)$ mode. The aperture radius is $5 \mu \mathrm{m}$.

where $n_{i}$ is the intrinsic carrier concentration. The resulting perimeter current for this device is

$$
I_{p}=2 \pi r_{\left(N_{\text {edge }}\right)} H_{0} \exp \left(\frac{V_{m\left(N_{\text {edge }}\right)}}{n k T}\right)
$$

where $H_{0}=q S d n_{i}, r_{\text {(Nedge) }}$ is the radius to the edge of the VCSEL junction, and the ideality factor $n$ is measured to be 1.907 for our devices. This value is about the same for oxidedefined or proton-implanted 850-nm VCSELs [8] because the quasi-Fermi level is always pinned at the perimeter of an Al$\mathrm{GaAs} / \mathrm{GaAs}$ junction, causing an $n k T$ current at the junction perimeter.

\section{Stimulated Current}

In addition to spontaneous recombination, radiative recombination includes stimulated recombination. The oxide aperture provides optical confinement for the transverse modes [15]. The optical waveguide is a weakly index-guided structure $\left(\Delta n \sim 3 \times 10^{-2}\right)$ and can be described by linear polarized $\left(\mathrm{LP}_{m l}\right)$ modes [16], [17]

$$
E(r)=\alpha \begin{cases}\frac{J_{m}\left(\frac{u r}{r_{\mathrm{a}}}\right)}{J_{m}(u)}, & r \leq r_{\mathrm{a}} \\ \frac{K_{m}\left(\frac{w r}{r_{\mathrm{a}}}\right)}{K_{m}(w)}, & r>r_{\mathrm{a}}\end{cases}
$$

where $\alpha$ is a normalization constant, $r_{a}$ is the oxide aperture radius, $J_{m}$ and $K_{m}$ are the Bessel function and the modified Hankel function of order $m$, respectively, $w$ are the eigenvalue solutions of the optical waveguide with the characteristic equation [18]

$$
\frac{u J_{m-1}(u)}{J_{m}(u)}=-\frac{w K_{m-1}(w)}{K_{m}(w)}
$$

The cutoff value for $u$ of the $\mathrm{LP}_{m l}$ mode is the $l$ th root of $J_{m 1}(\mathrm{u})$. Fig. 4 shows the field intensity for $\mathrm{LP}_{01}$ and $\mathrm{LP}_{11}$ for a VCSEL with a 10- $\mu$ m-diameter oxide aperture.

In this iterative model, we consider two LP modes, $\mathrm{LP}_{01}$ and $\mathrm{LP}_{11}$. A modal power ratio $\xi$ describes the ratio of the power in the $\mathrm{LP}_{11}$ mode to the sum of the powers in the $\mathrm{LP}_{11}$ and $\mathrm{LP}_{01}$ 
modes. The normalized power intensity distribution $\Pi_{k(i)}$ along the radial direction is

$$
\Pi_{k(i)}=\frac{\Re_{k}}{H_{m l}} \begin{cases}{\left[\frac{J_{m}\left(\frac{u_{m} l^{r}(i)}{r_{\mathrm{a}}}\right)}{J_{m}\left(u_{m l}\right)}\right]^{2},} & i \leq N_{\text {aperture }} \\ {\left[\frac{K_{m}\left(\frac{w_{m l^{r}(i)}}{r_{\mathrm{a}}}\right)}{K_{m}\left(w_{m l}\right)}\right]^{2},} & i>N_{\text {aperture }}\end{cases}
$$

where $\Re_{k}$ is the ratio of the power in the $k$ th mode to the total optical power, and $H_{m l}$ is the normalized maximum power for each mode. The fundamental $\mathrm{LP}_{01}$ mode and the next higher order mode $\left(\mathrm{LP}_{11}\right)$ have values of $\Re_{0}=1-\xi$ and $\Re_{1}=\xi$. Assuming radial symmetry, the total normalized power for each element $P_{k(i)}$ in Fig. 2 is

$$
P_{k(i)}=\Pi_{k(i)} A_{(i)}
$$

Above threshold, the stimulated current for each $k$ th mode $I_{\text {st_k(i) }}$ is proportional to the optical intensity and material gain $G$ and is given by

$$
I_{\mathrm{st}_{-}(i)}=P_{k(i)} G_{(i)} S_{k}
$$

where

$$
\begin{array}{r}
G_{(i)}=g_{0} \ln \left(\frac{J_{\mathrm{sp}(i)}}{J_{\mathrm{tr}}}\right) \\
S_{k}=\frac{\Re_{k} I_{\mathrm{std}}}{g_{\mathrm{th}} \sum_{i=1}^{N_{\text {edge }}} P_{k(i)}}
\end{array}
$$

where $g_{0}$ is the gain coefficient, and $J_{\mathrm{tr}}$ is the transparency current. $S_{k}$ is a seed factor and is used to determine the stimulated current for the $k$ th mode. The total stimulated current is $I_{\text {std }}$. The threshold gain $g_{\text {th }}$ is

$$
g_{\mathrm{th}}=\frac{1}{2 \Gamma_{\mathrm{r}} N_{\mathrm{w}} L_{\mathrm{w}}} \ln \left(\frac{1}{R_{\mathrm{n}} R_{p}}\right)
$$

where $\Gamma_{\mathrm{r}}$ is the relative QW confinement factor [19], $N_{\mathrm{w}}$ is the number of QWs, $L_{\mathrm{w}}$ is the width of each QW, and $R_{\mathrm{n}}$ and $R_{p}$ are the intensity reflectivities of the $n$ and $p$ DBRs. Note that $R_{\mathrm{n}}$ and $R_{p}$ and the mirror transmissivities $T_{\mathrm{n}}$ and $T_{p}$ are the product of amplitude reflectivities or amplitude transmissivities and their complex conjugates. These amplitude reflectivities and transmissivities are calculated using complex indexes for the DBR layers that account for the doping of the layers, so the DBR mirror losses are included in (27). For each mirror, $T+R+A=$ 1 , where $A$ is the fraction of light absorbed in the DBR mirror. In practice, the calculation of the reflectivity of the $\mathrm{p}-\mathrm{DBR}$ mirror includes all of the layers from the center of the QWs to the top layer of the p-DBR mirror and the reflectivity of the n-DBR mirror includes all of the layers from the center of the QWs to the bottom layer of the n-DBR mirror. Therefore, all losses (internal and mirror) are accounted for in (27). In edge-emitting lasers, $T+R=1$ and the internal losses of the long $(>200 \mu \mathrm{m})$ cavity appear explicitly in the threshold equation [20].

The higher order mode generally has a higher threshold gain than the fundamental mode because of the intensity profile overlap with the current distribution [21].

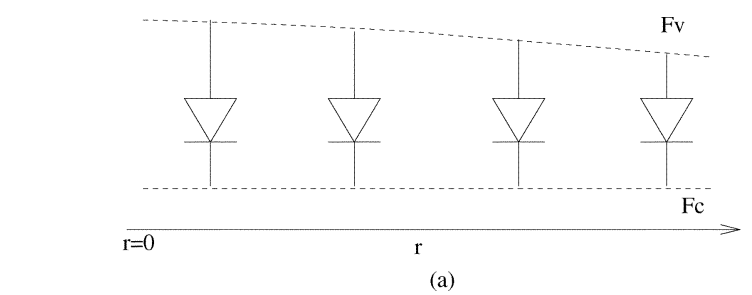

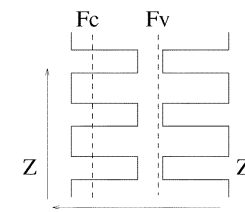

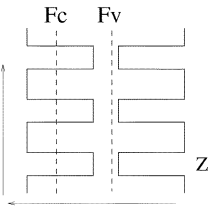

$\mathrm{E}$

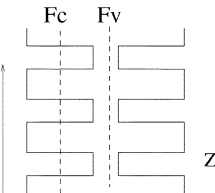

E

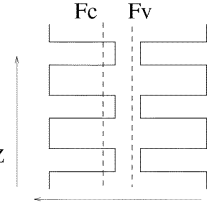

E (b)

Fig. 5. (a) Diodes and quasi-Fermi levels in the aperture area along the radial direction at $I=I_{\mathrm{th}}$. (b) Illustrations of the band diagram of the three QWs for each annular element in the cavity area. Each diode represents one annular element with three identical QWs. The dashed lines are the quasi-Fermi levels for electrons and holes.

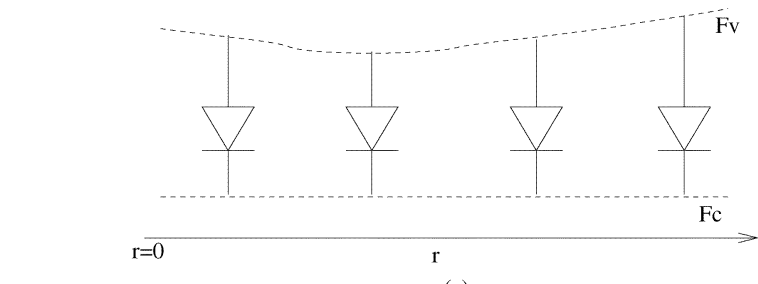

(a)

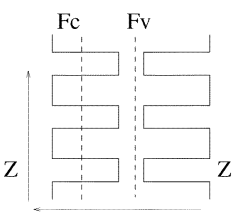

$\mathrm{E}$

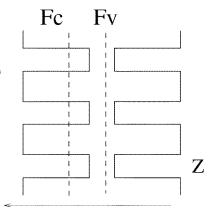

$\mathrm{E}$

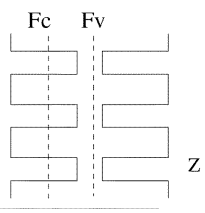

$\mathrm{E}$

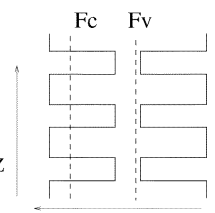

(b)

Fig. 6. (a) Diodes and quasi-Fermi levels in the aperture area along the radial direction at $I=3 I_{\mathrm{th}}$. (b) Illustrations of the band diagram of the three QWs for each annular element in the cavity area. Each diode represents one annular element with three identical QWs. The dashed lines are the quasi-Fermi levels for electrons and holes.

\section{E. Lateral Drift and Diffusion Currents}

The nonuniform carrier concentrations in the lateral direction and the voltage variation at the top node of each diode in Fig. 2 must be determined. Figs. 5(a) and 6(a) illustrate the variation of the hole and electron quasi-Fermi levels in the radial direction [22] at threshold and at three times threshold. One set of three QWs in Figs. 5(b) and 6(b) corresponds to one diode in Figs. 5(a) and 6(a). The diodes in Figs. 5(a) and 6(a) are shown only to illustrate that the quasi-Fermi-level separation varies from one annular element to another. Only four diodes are shown in Figs. 5(a) and 6(a). However, the typical number of annular regions used in the iterative model is 46 (32 for $r<r_{\text {(Naperture) }}$ and 14 for $r>r_{\text {(Naperture) }}$ ). Each diode in Figs. 2, 5, and 6 represents three identical QWs.

Near threshold (see Fig. 5), the gain is highest in the center $(r=0)$ and falls off gradually with increasing $r$. Therefore, the separation of the quasi-Fermi levels is greatest at $r=0$ and decreases with increasing radius. Above threshold (see Fig. 6), the 
high intensity of the fundamental mode nonuniformly depletes the carriers and reduces the gain for $r<\sim 3 \mu \mathrm{m}$. As a result, the separation of the quasi-Fermi levels can be reduced near $r=0$, reduced more for $r<3 \mu \mathrm{m}$, and then increased for $r>3 \mu \mathrm{m}$.

Since we assume that the n-DBR is an equipotential surface, the potential of the bottom nodes of all the diodes in Figs. 2, 5, and 6 are equal, and therefore the quasi-Fermi level in the conduction band is constant. Therefore, the lateral voltage variation results in a laterally varying quasi-Fermi level in the valance band as shown in Figs. 5(b) and 6(b). The lateral variation of the separation of the quasi-Fermi levels in Figs. 5 and 6 are associated with the nonuniform gain distributions discussed in Section III and shown in Fig. 12.

An ambipolar diffusion (AM) model is often used to calculate drift and diffusion of electrons and holes. The ambipolar diffusion coefficient $D_{\mathrm{a}}$ can be written as [23]

$$
D_{\mathrm{a}}=\frac{\mu_{p} D_{\mathrm{n}}+\mu_{\mathrm{n}} D_{p}}{\mu_{\mathrm{n}}+\mu_{p}}
$$

where $\mu_{\mathrm{n}}$ and $\mu_{p}$ are the electron and hole mobilities, and $D_{\mathrm{n}}$ and $D_{p}$ are the diffusion coefficients for the electrons and holes, respectively.

Since our iterative model assumes that the n-DBR is an equipotential surface, our model only accounts for hole drift and diffusion. By integrating the hole diffusion current density $\left(J_{\text {diff }}=-q D_{p} d p / d r\right)$ over the radius, we can find the diffusion current between adjacent diodes as

$$
I_{\operatorname{diff}(i)}=\frac{q D_{p} d\left(p_{(i)}-p_{(i-1)}\right)}{S_{(i)}} .
$$

SHB due to the resistance of the p-DBR mirror results in an external electrical field in the lateral plane, which causes hole drift in the QWs between adjacent diodes. The hole current density due to drift is related to the potential difference between the diodes and is given by $J_{\mathrm{drift}}=q \mu_{p} p d V / d r$. Taking the average hole concentration between adjacent diodes and integrating over the radius, the drift current is

$$
I_{\operatorname{drift}(i)}=\frac{q \mu_{p} d\left(p_{(i)}+p_{(i-1)}\right)\left(V_{m(i)}-V_{m(i-1)}\right)}{2 S_{(i)}} .
$$

The lateral drift-diffusion current between two adjacent nodes is the sum of the drift and diffusion current given by (29) and (30).

\section{F. Complete Model}

From the above results, we can set up a system of nodal equations (see the Appendix ) for the circuit in Fig. 2. Starting with an initial guess of the node voltages, a Newton-Raphson method solves the nonlinear system of equations to find the middle and top node voltages. After finding the node voltages, the resulting stimulated current must satisfy the round trip gain condition [(24)-(26)] and converge to the stimulated current $I_{\text {std }}$. The convergence condition is

$$
\sum_{k, i} I_{\text {st_k } k(i)}=I_{\text {std }} .
$$

If the convergence condition is not fulfilled, we need to adjust the bias voltage applied to the anode and the modal power ratio $\xi$. A flowchart describing the program is shown in Fig. 7. The

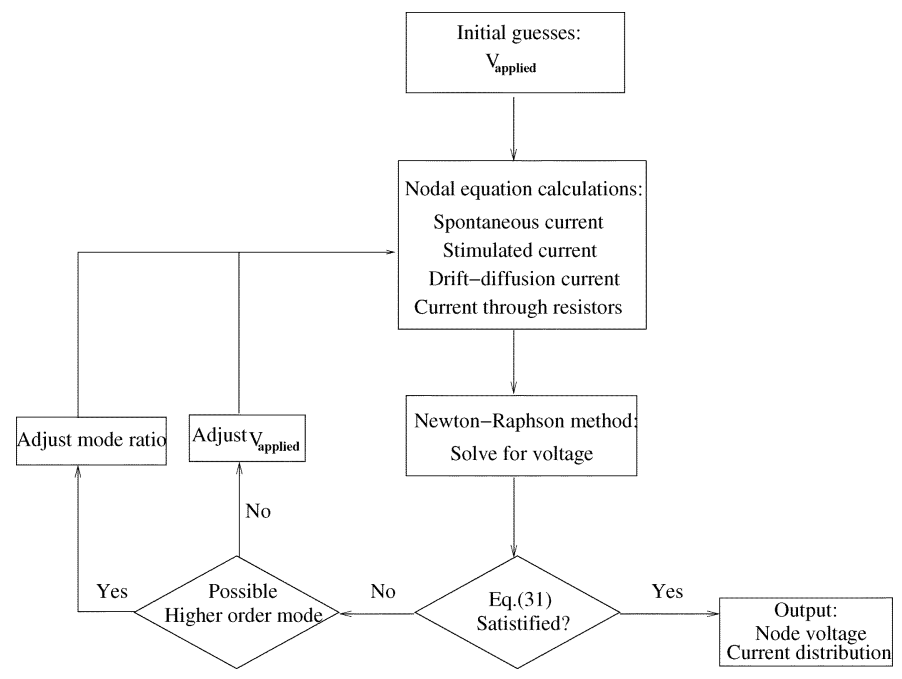

Fig. 7. Flowchart for the iterative model.

total current through the VCSEL is the sum of the stimulated, surface recombination, and spontaneous current. The threshold current can be found by making the stimulated current small $(\sim$ $0.01 \mathrm{~mA}$ ) while simultaneously satisfying (24)-(26) and (31). The $I-V$ curve can be obtained by iteratively increasing the input current.

\section{G. Lateral Carrier Transport Discussion}

We have the option of calculating the lateral current distribution by either the ambipolar (AM) or the drift and diffusion (DD) method using the parameters shown in Table II. The lateral carrier transport current simulated by the AM method uses (28) for the diffusion coefficient in (29). Fig. 8 shows the calculated $I-V$ curves using both the AM and the DD methods. The DD method has a 7\% higher threshold current than the AM method as shown in Table III.

The higher threshold current calculated by the DD method is due to higher current under the oxide and higher perimeter current. Although the DD method is more accurate, there is only $\sim 7 \%$ error in the calculation of the onset of the higher order mode calculated by the AM method. This small error is a bit surprising, since the AM method results in a lateral conductance that is about $50 \%$ of that calculated by the DD method for a stimulated current of $2 \mathrm{~mA}$, as shown in Fig. 9. The AM method underestimates the total charge transferred in the QW region, especially under the oxide region.

\section{H. Lateral Bandgap Renormalization}

Because the carrier injection varies laterally, the amount of bandgap renormalization varies laterally. The empirical bandgap energy reduction, $\Delta V_{\mathrm{bgr}}$, is related to the 2-D carrier concentrations by [24]

$$
\Delta V_{\mathrm{bgr}}=-3.2 \times 10^{-3} n_{\mathrm{s}}^{1 / 3}
$$

where $n_{\mathrm{s}}$ is the sheet carrier concentration. The reduction of the junction voltage by the carrier concentration is included in the iterative model by adding a dependent voltage source above each diode as shown in Fig. 10(a). The middle nodes (which 
TABLE II

VALUes of Theoretical PARAMETERS USED IN THE ITERATIVE MODEL

\begin{tabular}{|c|c|c|c|}
\hline Symbol & Parameters & Units & Value \\
\hline $\bar{B}$ & $\begin{array}{l}\text { bimolecular radiative } \\
\text { recombination constant }\end{array}$ & $\mathrm{cm}^{3} / \mathrm{s}$ & $2.5 \times 10^{-10}$ \\
\hline$S$ & surface recombination velocity & $\mathrm{cm} / \mathrm{s}$ & $1 \times 10^{5}$ \\
\hline$\mu_{\mathrm{p}}$ & hole mobility for GaAs & $\mathrm{cm}^{2} / \mathrm{V}-\mathrm{s}$ & 400 \\
\hline$g_{0}$ & gain coefficient & $\mathrm{cm}^{-1}$ & 880 \\
\hline$N_{\mathrm{w}}$ & number of quantum wells & - & 3 \\
\hline$L_{\mathrm{w}}$ & width of quantum well & $\AA$ & 70 \\
\hline$t_{\mathrm{p}}$ & p-DBR mirror thickness & $\mu \mathrm{m}$ & 3.5 \\
\hline$r_{\mathrm{a}}$ & aperture radius & $\mu \mathrm{m}$ & 5 \\
\hline$R_{\text {spdbr }}$ & p-DBR mirror sheet resistance & $\Omega / \square$ & $36,45,54$ \\
\hline$R_{\mathrm{so}}$ & $\begin{array}{l}\text { sheet resistance under the } \\
\text { oxide layer }\end{array}$ & $\Omega / \square$ & $\begin{array}{c}1000,3165 \\
5000\end{array}$ \\
\hline$n$ & $\begin{array}{l}\text { ideality factor for surface } \\
\text { recombination current }\end{array}$ & - & 1.907 \\
\hline$\gamma_{21}$ & $\begin{array}{l}\text { the ratio of the resistance of the } \\
\text { resistors in row two to that of } \\
\text { the resistors in row one }\end{array}$ & - & $2,3.5,5$ \\
\hline$\gamma_{\mathrm{vl}}$ & $\begin{array}{l}\text { anisotropy ratio between the } \\
\text { vertical and lateral resistivity }\end{array}$ & - & $4,6.8,8$ \\
\hline
\end{tabular}

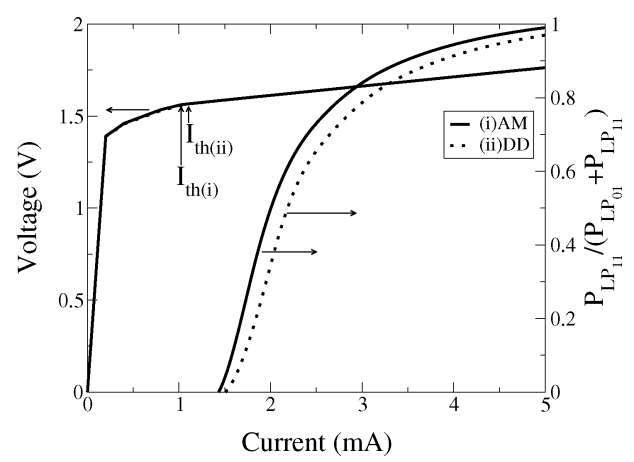

Fig. 8. $I-V$ curves and the ratio of the power in the $\mathrm{LP}_{11}$ mode (the first higher order mode) to the total laser power $\left(P_{\mathrm{LP01}}+P_{\mathrm{LP} 11}\right)$ as a function of injected current for the ambipolar (AM) and drift-diffusion (DD) method.

TABLE III

COMPARISON OF RESULTS OBTAINED BY EITHER THE AM OR THE DD METHOD

\begin{tabular}{llcc}
\hline \hline $\begin{array}{c}\text { Symbol } \\
\text { (Units) }\end{array}$ & \multicolumn{1}{c}{ Parameters } & AM & DD \\
\hline$I_{\mathrm{th}}(\mathrm{mA})$ & threshold current & 1.028 & 1.101 \\
$I_{\mathrm{p}}(\mathrm{mA})$ & perimeter current at threshold & 0.426 & 0.479 \\
$I_{\mathrm{ox}}(\mathrm{mA})$ & $\begin{array}{l}\text { current under oxide at threshold } \\
\Delta I(\mathrm{~mA})\end{array}$ & 0.599 & 0.674 \\
& $\begin{array}{l}\text { additional current above threshold } \\
\text { of the } \mathrm{LP}_{11} \text { mode required for } \\
\text { threshold of the } \mathrm{LP}_{01} \text { mode }\end{array}$ & 0.4 & 0.5 \\
\hline \hline
\end{tabular}

connect $\left.R_{2(i)} s\right)$ in Fig. 2 are supernodes consisting of the two nodes shown in Fig. 10(a). The voltage relation between these two nodes, $V_{\text {net(i) }}$ and $V_{m(i)}$ in Fig. 10(a) is given by

$$
V_{\text {net }(i)}=\Delta V_{\mathrm{bgr}}+V_{m(i)} \text {. }
$$

The system of equations for solving the network voltages and currents is shown in the Appendix. The voltage reduction due to bandgap renormalization $\left(\Delta V_{\mathrm{bgr}}\right)$ varies with the radius as shown in Fig. 10(b). In the cavity aperture, $\Delta V_{\mathrm{bgr}}$ depends on the amount of spatial hole burning. $\Delta V_{\mathrm{bgr}}$ rapidly becomes less negative under the oxide with increasing radius.

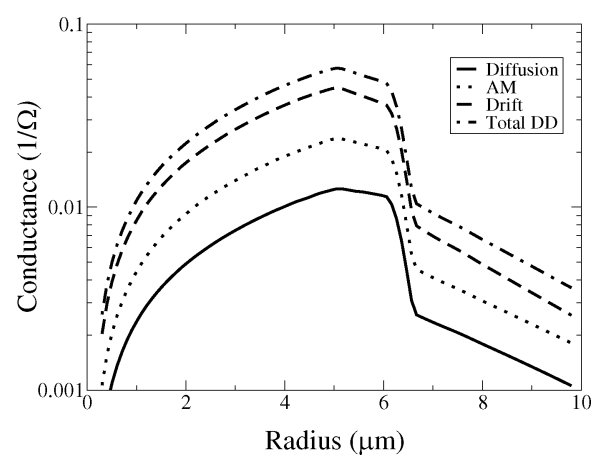

Fig. 9. Lateral conductance for the ambipolar method, diffusion only, drift only, and the sum of drift and diffusion currents at $I=3 I_{\mathrm{th}}$.
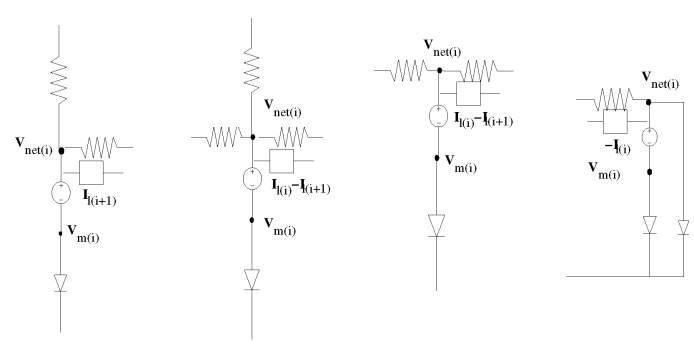

(a)

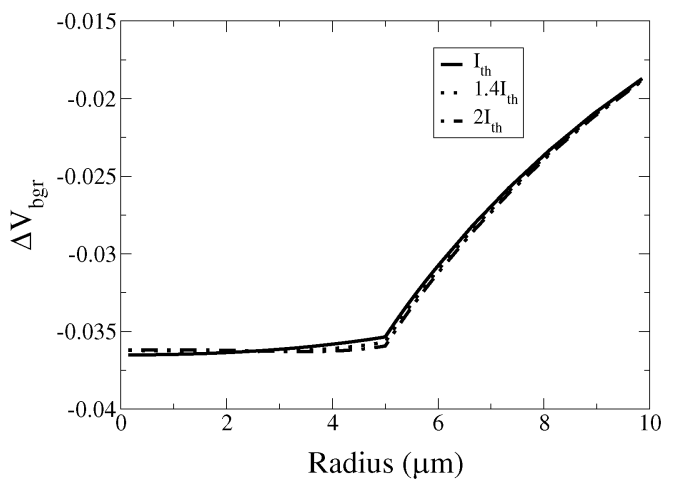

(b)

Fig. 10. (a) Bandgap renormalization modification for the middle nodes in Fig. 2. (b) Voltage variation resulting from bandgap renormalization as a function of radius under different current injection levels.

\section{Spatial Hole BuRning}

Here, we consider SHB in a VCSEL with an aperture radius of $5 \mu \mathrm{m}$ with the parameters described in Table II. The optical intensity profile of the $\mathrm{LP}_{01}$ (fundamental) and $\mathrm{LP}_{11}$ (next higher order mode) is initially determined by the cold cavity $(I=0$, $\left.V_{\text {applied }}=0\right)$ index profile. Figs. 11 and 12 illustrate the carrier concentration and gain distribution in the cavity aperture under different current injection levels, respectively. The local material gain distribution has the same trend and shape as the carrier concentration distribution. At low current values $\left(I=I_{\mathrm{th}}\right)$, the lateral gain has a distribution with a peak at $r=0$ and decreases monotonically with increasing $r$, as shown in Fig. 12. At threshold, the carrier concentration has the highest value in the center of the aperture, as does the local material gain profile. As a result, the fundamental mode (Fig. 4) is favored and becomes the first mode to lase. 


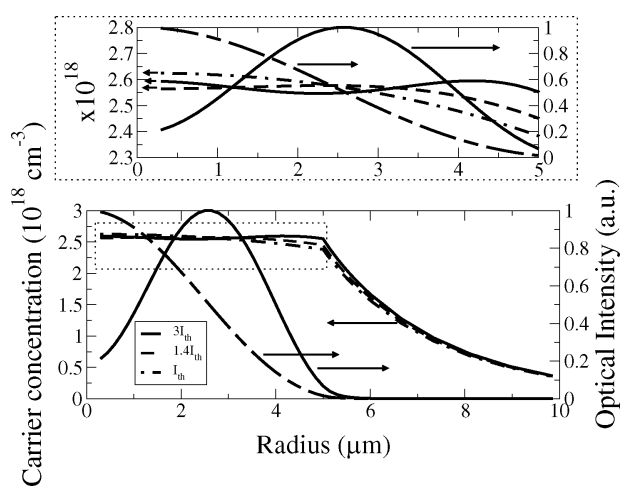

Fig. 11. Carrier concentration distributions and the optical intensity profiles of the fundamental and the first higher order mode as a function of radius. The dashed rectangle shows the carrier concentration for radius values of less than $5 \mu \mathrm{m}$ (i.e., inside the aperture region).

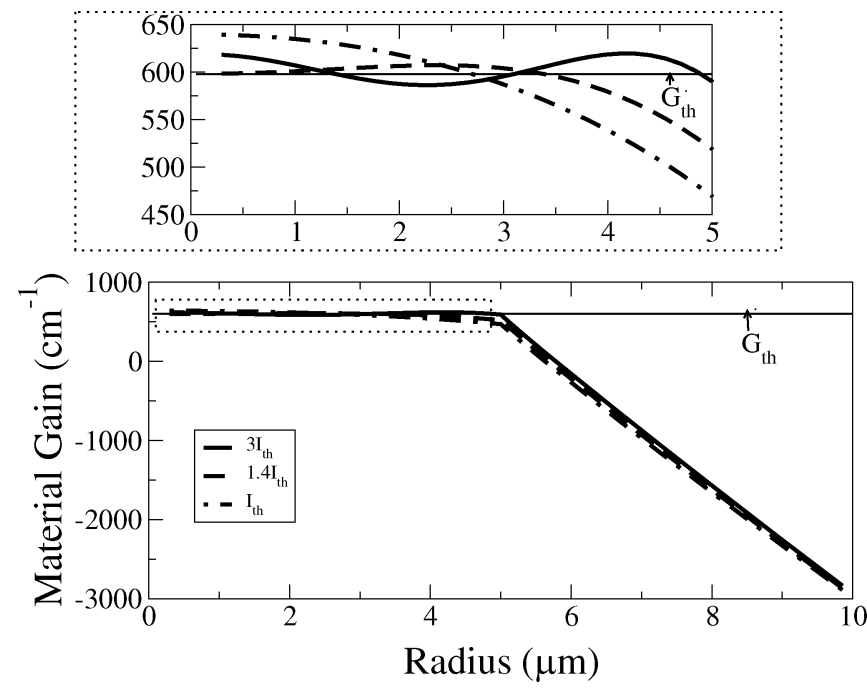

Fig. 12. Material gain as a function of radius for different values of current injection. The dashed rectangle shows the material gain for radius values of less than $5 \mu \mathrm{m}$ (i.e., inside the aperture region).

The carrier concentration becomes more nonuniform within the VCSEL aperture than outside the aperture with increasing current above threshold due to spatial hole burning [5]. As the current increases above threshold $\left(I=1.4 I_{\mathrm{th}}\right)$, the increase in the fundamental mode intensity near $r=0$ causes an increase in stimulated emission in the vicinity of $r=0$, which reduces the number of carriers available to provide gain (this process is called hole burning [25]). This results in a flattening of the carrier distribution within the aperture and a substantial increase in the carriers in the vicinity of $r \sim 3 \mu \mathrm{m}$ (the location of the peak of the $\mathrm{LP}_{11}$ mode), since the total modal gain must remain constant. With further increases in the current $\left(I=3 I_{\mathrm{th}}\right)$, the carrier concentration (and therefore the gain) in the center of the aperture is further reduced and the resulting gain profile has a favorable overlap with the $\mathrm{LP}_{11}$ mode. Thus, the gain becomes substantial in the vicinity of the peak of the $\mathrm{LP}_{11}$ mode, and this higher order mode begins to produce noticeable power. As the intensity of the $\mathrm{LP}_{11}$ mode increases, there is less power in the $\mathrm{LP}_{01}$ mode, which allows the gain and carrier concentrations near $r=0$ to increase as shown in Figs. 11 and 12. In

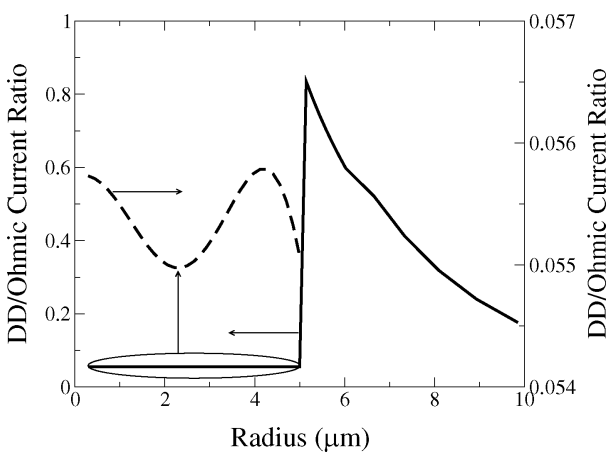

Fig. 13. Ratio of lateral DD current to Ohmic current along the radial direction for $I=3 I_{\mathrm{th}}$. The dashed inset shows the structure of the DD/Ohmic current ratio for $r<5 \mu \mathrm{m}$ on an expanded scale.

general, the presence of spatial hole burning requires that the quasi-Fermi levels are not pinned at threshold. Therefore, the spontaneous current and spontaneous emission continues to increase above threshold. However, the round trip gain of each steady-state lasing mode has to equal 1 . The variation of the separation of the quasi-Fermal levels due to spatial hole burning for the examples shown in Fig. 12 for $I=I_{\text {th }}$ and $I=3 I_{\text {th }}$ are shown in Figs. 5 and 6.

\section{RESULTS}

\section{A. Calculated Results}

We perform a sample simulation with the parameters in Table II and apply the DD method with a bandgap renormalization correction at an injection level equal to $3 I_{\text {th }}$. The Ohmic current flowing through the resistors $\left(R_{1(i)}, R_{v(i)}\right.$, and $\left.R_{2(i)}\right)$ will always be larger than the lateral DD current flowing in the QWs $\left(I_{1(i)}\right)$. Within the aperture, the Ohmic current is about 18 times larger than DD current as shown in Fig. 13. At $I=3 I_{\mathrm{th}}$, the $\mathrm{LP}_{11}$ mode is dominant in the cavity region. As a result of spatial hole burning, the $\mathrm{DD} / \mathrm{Ohmic}$ current ratio as a function of radius has low values for $r<5 \mu \mathrm{m}$ and high values for $r>5 \mu \mathrm{m}$. The dip of the DD/Ohmic ratio for $r<5 \mu \mathrm{m}$ from the maximum to minimum is about $13 \%$, which tracks the changes of carrier concentration as shown in Fig. 11. Under the oxide region, the lateral drift current in the $\mathrm{QW}$ region becomes important.

Fig. 14(a) shows the current density distribution in the QW at $I=3 I_{\mathrm{th}}$. The stimulated current is the main contribution to the total current. The lateral Ohmic current density near the top of the p-DBR mirror (flowing in the resistors of row one) is shown by the solid line, and the lateral Ohmic current density near the bottom of the p-DBR mirror (flowing in the resistors of row two) is shown by the dotted line in Fig. 14(b). A positive lateral current density means that the voltage on the node with a larger radius is higher than the adjacent node with a smaller radius. In this case, the current flows towards the center of the aperture. The current density flow from the top to the bottom of the p-DBR mirror is shown in Fig. 14(c).

Applying a voltage ( $V_{\text {applied }}$ ) at the node labeled "anode" results in a nonuniform current density around the edge of the aperture as shown in Fig. 14. Within the aperture, the middle nodes carry current from both vertical and horizontal resistors 


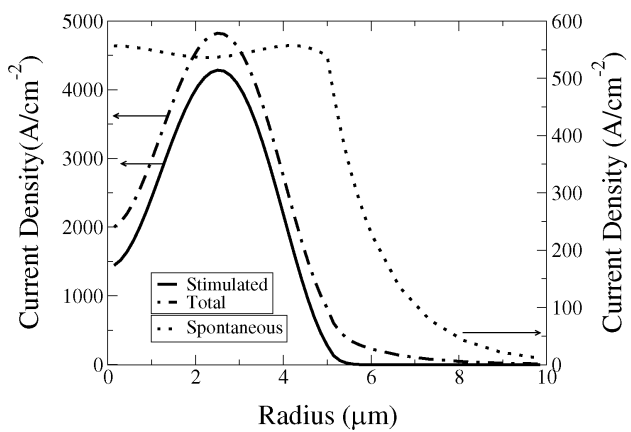

(a)

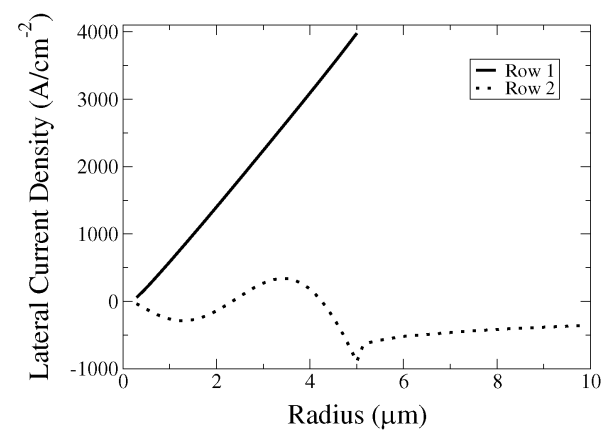

(b)

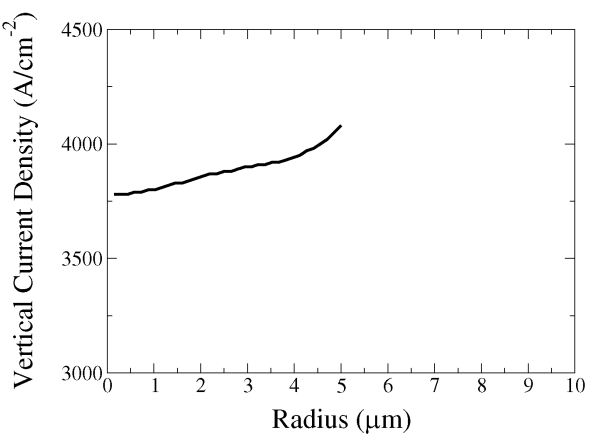

(c)

Fig. 14. Current density as a function of radius for (a) spontaneous, stimulated, and total current, (b) the lateral Ohmic current density from the resistors in row one and resistors in row two in Fig. 2, and (c) the vertical Ohmic current density from the vertical resistors. All plots are for $I=3 I_{\mathrm{th}}$.

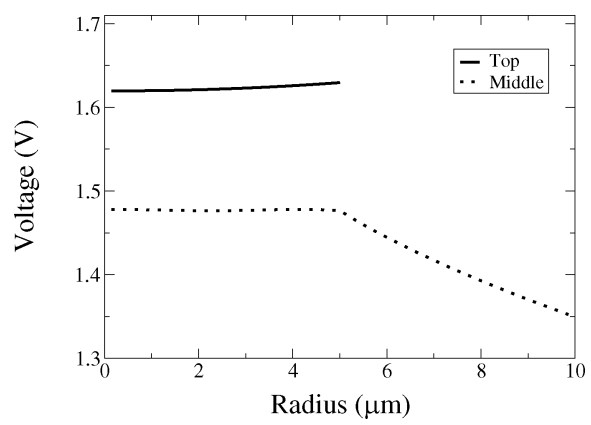

Fig. 15. Voltage distribution in the top and middle nodes for $I=3 I_{\mathrm{th}}$.

to the diodes. However, at the edge of the aperture, the middle nodes only carry current radially and downwards to the diodes.

Fig. 15 shows the voltage distribution of the top and middle nodes. The voltage along the top nodes decreases towards the middle of the aperture since the Ohmic contact is near the edge

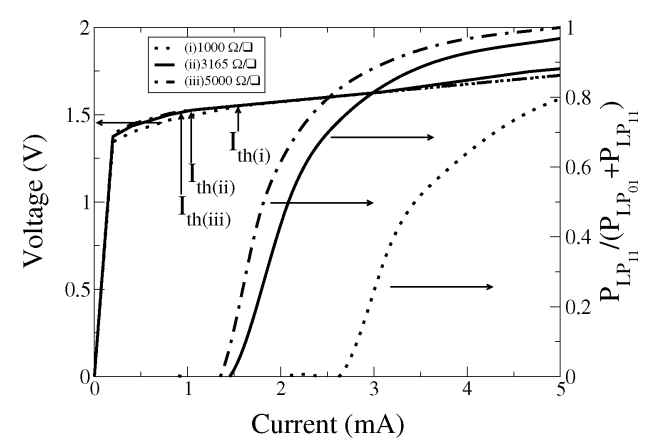

Fig. 16. $I-V$ curves and the ratio of the power in the $\mathrm{LP}_{11}$ mode (the first higher order mode) to the total laser power $\left(P_{\mathrm{LP} 01}+P_{\mathrm{LP} 11}\right)$ as a function of injected current for different sheet resistances under the oxide layers.

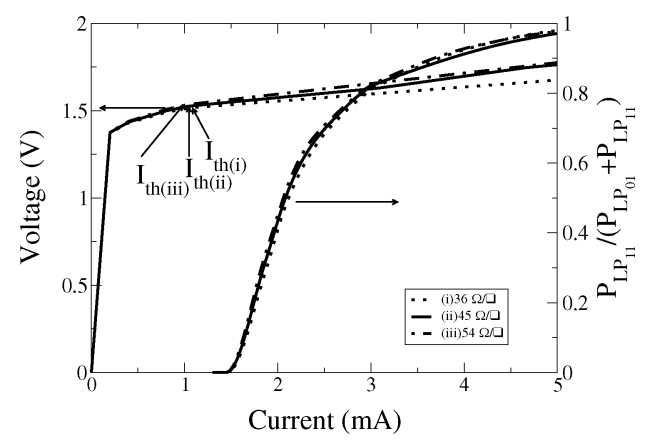

Fig. 17. $I-V$ curves and the ratio of the power in the $\mathrm{LP}_{11}$ mode (the first higher order mode) to the total laser power $\left(P_{\mathrm{LP} 01}+P_{\mathrm{LP} 11}\right)$ as a function of injected current for different p-DBR sheet resistances.

of the aperture. The voltage of the middle nodes is reasonably flat within the aperture and decreases with increasing radius outside the aperture.

Calculated $I-V$ curves for sheet resistance variations under the oxide layer and in the p-DBR regions for $\gamma_{21}=3.5$ and $\gamma_{\mathrm{vl}}=6.8$ are shown in Figs. 16 and 17, respectively. Increasing the sheet resistance under the oxide layer $R_{\text {so }}$ reduces the threshold current as shown in Fig. 16 since current at $r>5$ to $6 \mu \mathrm{m}$ has little overlap with the optical mode and contributes to perimeter current. These results show that the threshold current is reduced by $40 \%$ by increasing $R_{\text {so }}$ from 1000 to $5000 \Omega /$ square. The result is similar to that found for stripe-geometry double-heterostructure lasers [26]. The series resistance calculated from the $I-V$ curve is almost independent of $\mathrm{R}_{\mathrm{so}}$. Fig. 16 shows that a lower $R_{\text {so }}$ helps maintain single-mode operation to higher current values, at the expense of a higher single-mode threshold.

Changing the p-DBR mirror sheet resistance $R_{\text {spdbr }}$ has little effect on the onset of threshold of the next higher order mode, as shown in Fig. 17. Therefore, a lower $R_{\text {spdbr }}$ can be chosen to minimize the series resistance. The power ratio $\xi$ in Fig. 17, unlike $\xi$ in Fig. 16, varies less than $1 \%$.

Figs. 18 and 19 show how the adjustable parameters $\gamma_{21}$ and $\gamma_{\mathrm{vl}}$ affect the $I-V$ curves for $R_{\text {spdbr }}=45 \Omega /$ square and $R_{\text {so }}=$ $3165 \Omega$ /square. The series resistance changes as $\gamma_{\mathrm{vl}}$ changes, but $\xi$ and the threshold current remain almost unchanged. Increasing $\gamma_{\mathrm{vl}}$ means the resistances at the heterointerfaces and/or 


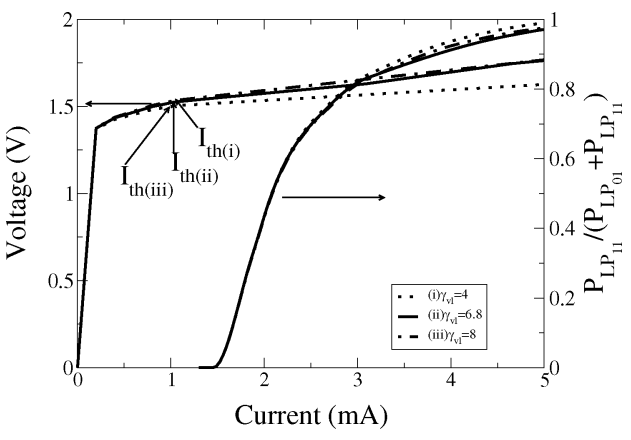

Fig. 18. $I-V$ curves and the ratio of the power in the $\mathrm{LP}_{11}$ mode (the first higher order mode) to the total laser power $\left(P_{\mathrm{LP} 01}+P_{\mathrm{LP} 11}\right)$ as a function of injected current for different vertical to lateral resistivity ratios.

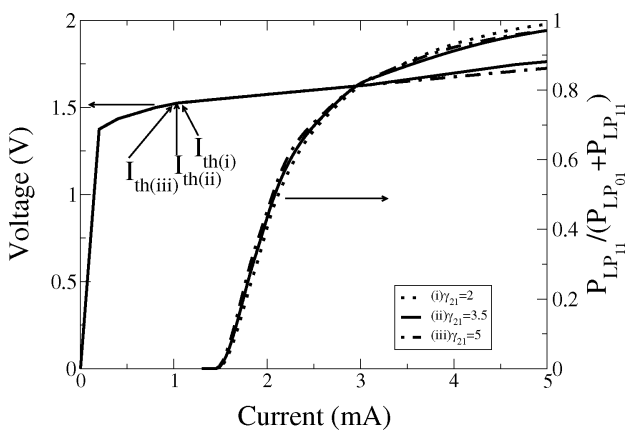

Fig. 19. $I-V$ curves and ratio of the power in the $\mathrm{LP}_{11}$ mode (the first higher order mode) to the total laser power $\left(P_{\mathrm{LP} 01}+P_{\mathrm{LP} 11}\right)$ as a function of injected current for different resistance ratios of resistors in row two to row one.

TABLE IV

VALUES OF THE ADJUSTABLE RESISTIVITY ANISOTROPY PARAMETERS $\gamma_{21}$ AND $\gamma_{\mathrm{vl}}$ THAT BEST MATCH EXPERIMENTAL RESULTS

\begin{tabular}{lllc}
\hline \hline Symbol & \multicolumn{1}{c}{ Parameters } & Units & Value \\
\hline$\gamma_{21}$ & $\begin{array}{l}\text { ratio of the resistance of the } \\
\text { resistors in row two to that of } \\
\text { the resistors in row one } \\
\text { anisotropy ratio between the } \\
\text { vertical and lateral resistivity }\end{array}$ & 3.5 \\
$\gamma_{\mathrm{ll}}$ & 7.7 \\
\hline \hline
\end{tabular}

the layer resistances in the p-DBR mirror are higher, resulting in a higher series resistance as shown in Fig. 18.

On the other hand, the threshold current, series resistance and $\xi$ dependence on $\gamma_{21}$ are minor as $\gamma_{21}$ changes from 2 to 5 , as shown in Fig. 19.

In summary, the variation of $R_{\mathrm{so}}$ has the most impact on both threshold current and on maintaining single-mode operation at higher current levels. $R_{\mathrm{spdbr}}$ and $\gamma_{\mathrm{vl}}$ play a major role in determining the series resistance of the device.

\section{B. Comparison With Experiment}

Table IV shows the values of the adjustable resistivity anisotropy parameters $\gamma_{21}$ and $\gamma_{\mathrm{vl}}$ that closely match the theoretical to the measured $I-V$ and $L-I$ characteristics shown in Fig. 20 for a multimode VCSEL with an aperture radius of $5 \mu \mathrm{m}$. Adjusting $\gamma_{\mathrm{vl}}$ mainly effects the slope of the series resistance (see Fig. 18).

The measurements were done under $\mathrm{CW}$ wave operation at current levels below thermal rollover. The measured voltage

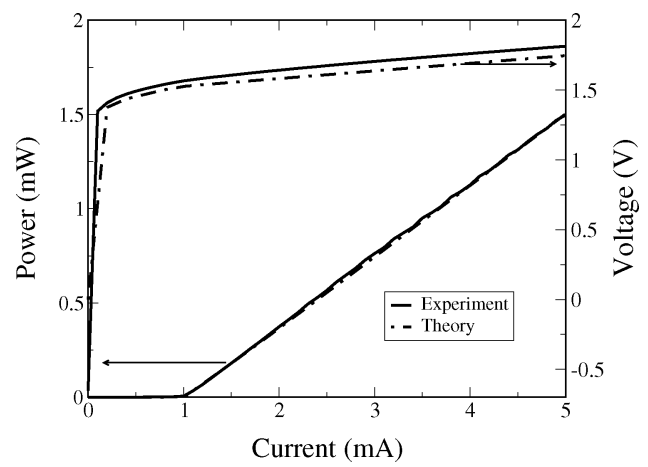

Fig. 20. Comparison of theoretical and experimental $I-V$ and $L-I$ curves.

(Fig. 20) is higher than that calculated with this model. This difference cannot be eliminated by adding more resistance at the hetero-interfaces since the slope of the resistance is determined by $\gamma_{\mathrm{vl}}$. Adding more resistance for the hetero-interfaces will change the differential series resistance.

The vertical resistivity is made up of the resistivity of the $15 \% \mathrm{AlGaAs}$ layers, the $85 \% \mathrm{AlGaAs}$ layers, and the effective resistivity of the hetero-interfaces. Each mirror layer has two opposed hetero-interfaces, so each pair of hetero-interfaces has one "forward" biased and one "reverse" biased junction, each with a small offset voltage. The difference between the measured and modeled $I-V$ is the sum of all of the nonlinear voltage drops for each mirror period.

For the example in this paper, the model requires an anisotropy ratio $\gamma_{\mathrm{vl}}$ of 7.7 to match the experimental results, suggesting that only $16 \%$ of the vertical resistance results from the conductivity in the doped mirror layers in the p-DBR mirror. The remainder of the vertical resistance is due to the small voltage drops at the hetero-interfaces.

The difference between the calculated and measured voltages (about 5\%) above threshold can be described by the following polynomial expression

$$
\Delta V=0.0008 I^{3}-0.0105 I^{2}+0.0453 I+0.0048 .
$$

The optical output power $P$ is obtained from the stimulated current

$$
P=\eta_{\mathrm{s}} \frac{I_{\mathrm{st}}}{\eta_{\mathrm{st}}}
$$

where $\eta_{\mathrm{st}}$ is the stimulated quantum efficiency and can be expressed as the ratio of the stimulated current to the difference between the total current $I_{\text {total }}$ and the threshold current $I_{\text {th }}$

$$
\eta_{\mathrm{st}}=\frac{I_{\mathrm{st}}}{I_{\mathrm{total}}-I_{\mathrm{th}}} .
$$

The slope of the $L-I$ curve $\eta_{\mathrm{s}}$ is

$$
\eta_{\mathrm{s}}=\frac{E_{\phi} \eta_{\mathrm{st}} T_{p}}{2-R_{p}-R_{\mathrm{n}}}
$$

where $E_{\phi}$ is the photon energy, $T_{p}$ is the intensity transmissivity of the p-DBR mirror, and $R_{p}$ and $R_{\mathrm{n}}$ are the intensity reflectivities of the p-DBR and n-DBR mirrors [see the discussion associated with (27)]. 


$$
\begin{aligned}
& V_{\operatorname{top}(i)}= \begin{cases}V_{\text {applied }}, & \text { for } i=N_{\text {aperture }} \\
\frac{V_{\mathrm{top}(i+1)} * G_{1(i+1)}+V_{\mathrm{top}(i-1)} * G_{1(i)}+V_{m(i)} * G_{v(i)}}{G_{(1 i)}+G_{v(i)}+G_{1(i+1)}}, & \text { for } 1<i<N_{\text {aperture }} \\
\frac{V_{\mathrm{top}(i+1)} * G_{1(i+1)}+V_{m(i)} * G_{v(i)}}{G_{1(i+1)}+G_{v(i)}}, & \text { for } i=1\end{cases}
\end{aligned}
$$

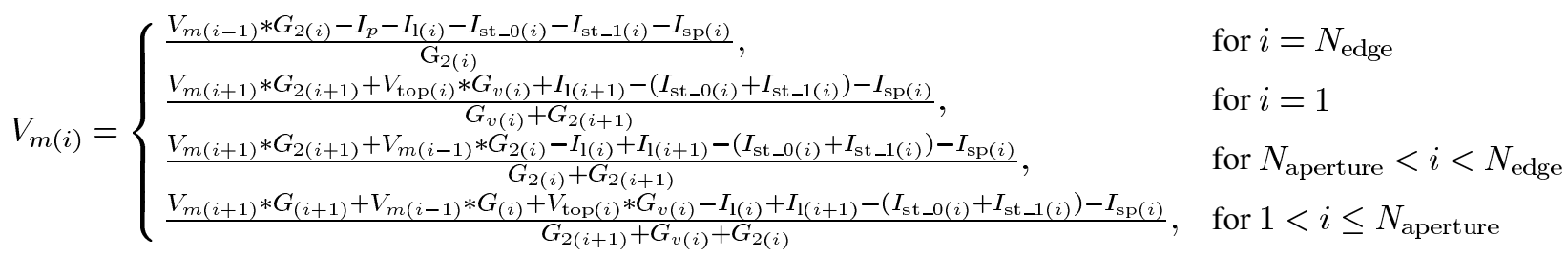

\section{CONCLUSION}

We demonstrate a SPICE-like approach to solve the detailed current and voltage distributions in VCSELs. The model includes both spontaneous and stimulated recombination in the quantum wells and includes surface recombination at the junction perimeter. Drift, diffusion, and bandgap renormalization are included in the model. SHB is dominated by the voltage drop due to the resistance of the p-DBR mirror moderated by lateral drift and diffusion of carriers. This model shows good agreement with experimentally measured $I-V$ and $L-I$ curves and provides insight into the detailed distributions of current and voltage within a VCSEL.

\section{APPENDIX}

We can find the voltage for each node in Fig. 2 from nodal analysis by Kirchhoff's current law. The top nodes are on top of the vertical resistors in Fig. 2. The top node voltages $V_{\text {top }(i)}$ are given by (A1), which is shown at the top of the page.

Similarly, the middle node voltages are given by (A2), shown at the top of the page, where $G$ is the conductance and $I_{1}$ is the sum of $I_{\text {drift }}$ and $I_{\text {diff. }} I_{\text {st_o }}(\mathrm{i})$ is the stimulated current for the $\mathrm{LP}_{01}$ mode and $I_{\text {st_ } 1}(\mathrm{i})$ is the stimulated current for the $\mathrm{LP}_{11}$ mode [see (24)] in the $i$ th annular region.

When we consider the bandgap renormalization effect, $V_{\text {net }(i)}$ in (33) replaces $V_{m(i)}$ in both (30) (for the drift current) and in (A2) for the system equation. Note that $V_{m(i)}$ in (33) are used to calculate the spontaneous current (16).

\section{ACKNOWLEDGMENT}

The authors would like to thank Dr. D. Bour (Photodigm) and Dr. P. Pinsukanjana (IntelliEPI) for comments and critical readings of the manuscript, and Dr. A. Nachman (AFOSR) and Mr. G. J. Ford for partial financial support.

\section{REFERENCES}

[1] J. J. Morikuni, P. V. Mena, A. V. Harton, K. W. Wyatt, and S. M. Kang, "Spatially independent VCSEL models for the simulation of diffusive turn-off transients," J. Lightw. Technol., vol. 17, no. 1, pp. 95-102, Jan. 1999.

[2] G. R. Hadley, K. L. Lear, M. E. Warren, K. D. Choquette, J. W. Scott, and S. W. Corzine, "Comprehensive numerical modeling of verticalcavity surface-emitting lasers," IEEE J. Quantum Electron., vol. 32, no. 4, pp. 607-616, Apr. 1996.
[3] H. K. Bissessur, F. Koyama, and K. Iga, "Modeling of oxide-confined vertical-cavity surface-emitting lasers," IEEE J. Sel. Topics Quantum Electron., vol. 3, no. 2, pp. 344-352, Mar./Apr. 1997.

[4] M. Streiff, A. Witzig, M. Pfeiffer, P. Royo, and W. Fichtner, "A comprehensive VCSEL device simulator," IEEE J. Sel. Topics Quantum Electron., vol. 9, no. 3, pp. 879-891, May/Jun. 2003.

[5] M. Osiński, V. A. Smagley, M. Lu, G. A. Smolyakov, P. G. Eliseev, B. P. Riely, P. H. Shen, and G. J. Simonis, "Self-consistent calculation of current self-distribution effect in GaAs-AlGaAs oxide-confined VCSELs," IEEE J. Sel. Topics Quantum Electron., vol. 9, no. 5, pp. 1422-1430, Sep./Oct. 2003.

[6] R. Michalzik and K. J. Ebeling, "Modeling and design of proton-implanted ultralow-threshold vertical-cavity laser diodes," IEEE J. Quantum Electron., vol. 29, no. 6, pp. 1963-1974, Jun. 1993.

[7] M. Achtenhagen, A. A. Hardy, and E. Kapon, "Threshold analysis of vertical-cavity surface-emitting lasers with intracavity contacts," IEEE J. Quantum Electron., vol. 42, no. 4, pp. 891-897, Apr. 2006.

[8] A. Ramaswamy, J. P. van der Ziel, J. R. Biard, R. Johnson, and J. A. Tatum, "Electrical characteristics of proton-implanted vertical-cavity surface-emitting lasers," IEEE J. Quantum Electron., vol. 34, no. 11, pp. 2233-2240, Nov. 1998.

[9] G. Bourdon, I. Robert, I. Sagnes, and I. Abram, "Spontaneous emission in highly excited semiconductors: Saturation of the radiative recombination rate," J. Appl. Phys., vol. 92, no. 11, pp. 6595-6600, Dec. 2002.

[10] R. F. Nabiev and C. J. Chang-Hasnain, "Voltage drop in n- and p-type bragg reflectors for vertical-cavity surface-emitting lasers," IEEE Photon. Technol. Lett., vol. 7, no. 7, pp. 733-735, Jul. 1995.

[11] R. Olshansky, C. B. Su, J. Manning, and W. Powazinik, "Measurement of radiative and nonradiative recombination rates in InGaAsP and AlGaAs light sources," IEEE J. Quantum Electron., vol. QE-20, no. 8, pp. 838-854, Aug. 1984.

[12] G. P. Agrawal and N. K. Dutta, Semiconductor Lasers. New York: Van Nostrand Reinhold, 1993, ch. 2.

[13] S. L. Chuang, Physics of Optoelectronic Devices. New York: Wiley, 1995, ch. 9.

[14] C. H. Henry, R. A. Logan, and F. R. Merritt, "The effect of surface recombination on current in $\mathrm{Al}_{\mathrm{x}} \mathrm{Ga}_{1-\mathrm{x}}$ As heterojunctions," J. Appl. Phys., vol. 49, no. 6, pp. 3530-3542, Jun. 1978.

[15] P. Bienstman, R. Baets, J. Vukusic, A. Larsson, M. J. Noble, M. Brunner, K. Gulden, P. Debernardi, L. Fratta, G. P. Bava, H. Wenzel, B. Klein, O. Conradi, R. Pregla, S. A. Riyopoulos, J. P. Seurin, and S. L. Chuang, "Comparison of optical VCSEL models on the simulation of oxide-confined devices," IEEE J. Quantum Electron., vol. 37, no. 12, pp. 1618-1631, Dec. 2001.

[16] G. R. Hadley, "Effective index model for vertical-cavity surface-emitting lasers," Opt. Lett., vol. 20, no. 13, pp. 1483-1485, Jul. 1995.

[17] R. Michalzik and K. J. Ebeling, "Generalized BV diagrams for higher order transverse modes in planar vertical-cavity laser diodes," IEEE J. Quantum Electron., vol. 31, no. 8, pp. 1371-1379, Aug. 1995.

[18] K. Kawano and T. Kitoh, Introduction to Optical Waveguide Analysis. New York: Wiley, 2001, ch. 2.

[19] S. W. Corzine, R. S. Geels, J. W. Scott, R. Yan, and L. A. Coldren, "Design of Fabry-Perot surface-emitting lasers with a periodic gain structure," IEEE J. Quantum Electron., vol. 25, no. 6, pp. 1513-1524, Jun. 1993.

[20] J. R. Biard, W. N. Carr, and B. S. Reed, "Analysis of a GaAs laser," Trans. Metallurgic. Soc. AIME, vol. 230, pp. 286-290, 1964. 
[21] P. Bienstman, B. Demeulenaere, B. Dhoedt, and R. Basets, "Simulation results of transverse-optical confinement in airpost, regrown, and oxidized vertical-cavity surface-emitting laser structures," J. Opt. Soc. Amer. B, vol. 16, no. 11, pp. 2055-2059, Nov. 1999.

[22] W. B. Joyce, "Carrier transport in double-heterostructure active layers," J. Appl. Phys., vol. 53, no. 11, pp. 7235-7239, Nov. 1982.

[23] J. Buus and M. Danielsen, "Carrier diffusion and higher order transversal modes in spectral dynamics of the semiconductor laser," IEEE J. Quantum Electron., vol. QE-13, no. 8, pp. 669-674, Aug. 1977.

[24] E. D. Jones, M. Blount, W. Chow, H. Hou, J. A. Simmons, Y. Kim, and T. Schmiedel, "Bandgap renormalization: GaAs/AlGaAs quantum wells," Proc. SPIE, vol. 3283, pp. 350-356, 1998.

[25] H. Statz, C. L. Tang, and J. M. Lavine, "Spectral output of semiconductor lasers," J. Appl. Phys., vol. 35, no. 9, pp. 2581-2585, Sep. 1964.

[26] W. T. Tsang, "The effects of lateral current spreading, carrier outdiffusion, and optical mode losses on the threshold current density of GaAs- $\mathrm{Al}_{\mathrm{x}} \mathrm{Ga}_{1-\mathrm{x}}$ As stripe-geometry DH lasers," J. Appl. Phys., vol. 49, no. 3, pp. 1031-1044, Mar. 1978.

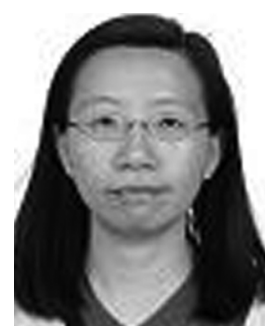

Hsueh-hua Chuang (S'03) was born in Kaohsiung, Taiwan, R.O.C. She received the B.S. degree in environmental engineering from National Chung-Hsing University, Taichung, Taiwan, R.O.C., in 1993, the M.S. degree in environmental engineering from Colorado State University, Fort Collins, in 1996, and the M.S. degree in computer science engineering from the University of Texas, Arlington, in 2001. She is currently working toward the Ph.D. degree in the Electrical Engineering Department, Southern Methodist University (SMU), Dallas, TX.

She is currently a Graduate Research Assistant and Teaching Assistant with the Electrical Engineering Department, Southern Methodist University (SMU), where she is involved with the simulation of vertical-cavity surface-emitting lasers (VCSELs) and the design, fabrication, and testing of compound semiconductor devices. Her research interests include long-wavelength VCSELs, photonic integrated circuits, optical sensors, and microelectromechanical system (MEMS) devices.

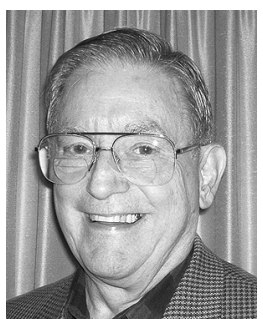

James R. Biard (S'56-M'67-F'68-LF'95) was born in Paris, TX. He received the B.S., M.S., and Ph.D. degrees from Texas A\&M University, College Station, in 1954, 1956, and 1957, respectively, all in electrical engineering.

He became Chief Scientist with the Honeywell MICRO SWITCH Division in 1987. He retired in December 1998 and was hired by Honeywell and Finisar as a consultant. In the Finisar job assignment, he is part of a team developing vertical-cavity surface-emitting lasers (VCSELs). He is also involved in the interface between the VCSEL team and universities. He started the MICRO SWITCH IC \& Sensor Design Center in Richardson, TX, and his product development responsibilities include optoelectronic components (light-emitting diodes and photo detectors), fiber-optic components and transmitter and receiver modules, and silicon Hall-effect and pressure sensors. $\mathrm{He}$ is also on the staff of Texas A\&M University as an Adjunct Professor of Electrical Engineering. He has served in this capacity since 1980. From 1978 to 1987, he was Chief Scientist of the Honeywell Optoelectronics Division and was a member of the Components Group Sensor Planning Team. He was also the Components Group representative on the Honeywell Technology Board (HTB). The HTB was concerned with the development and transfer of technology throughout the Honeywell corporate structure. He joined Spectronics, Inc., as Vice President of Research in 1969 when the company was founded. Honeywell acquired Spectronics, Inc. in 1978. Previously, he worked for Texas Instruments, Inc. (TI), from 1957 to 1969. While at TI he participated in the development of transistor circuits, microwave and optoelectronic components, avalanche photodiodes, silicon MOS technology, and compound semiconductor materials technology.

Dr. Biard is a member of the National Academy of Engineering. In 1989, he was the recipient of the Honeywell Lund Award. In 1986, he was recognized as a Distinguished Alumnus of Texas A\&M University. In 1985, he was a recipient of the Patrick E. Haggerty Innovation Award for his contribution to the design and development of Schottky Logic.

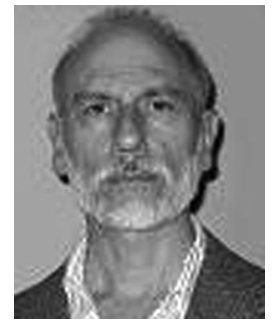

James Guenter (SM'06) received the B.S. degree from Rice University, Houston, TX, and the M.S. degree from the University of Texas at Dallas, both in physics.

While at Honeywell from 1979 to 1994 , he was involved with IR and visible LEDs for displays and for communications, high-resistivity silicon and compound semiconductor photodiodes and phototransistors, and various optoelectronic applications. Beginning in 1994, as he worked on the team transferring VCSEL technology from the corporate labs to the manufacturing division, he concentrated on assessing and improving VCSEL reliability and on developing components for the data communications markets. As a Senior Fellow during this time, and after the sale of the Honeywell VCSEL products division to Finisar in 2004, he also participated in intellectual property assessment and development and on VCSEL structures for new applications. He holds 30 U.S. patents, has authored or coauthored dozens of papers, and has served for many years on the committee for SPIE's Vertical-Cavity Surface-Emitting Lasers program, (and as conference chair for 2007 and 2008).

Mr. Guenter was the recipient of the H.W. Sweatt Award.

Ralph H. Johnson received the B.S. degree in physics from Texas A\&M University in 1978, the M.A. degree in physics from the University of California, Berkeley, in 1980, and the Ph.D. degree in electrical engineering from Texas A\&M University, College Station, in 1985.

He has worked in semiconductor device design and modeling, III-V epitaxy, process development and production for III-V's, and silicon sensors and integrated circuits. His epitaxy experience includes MBE (six years), MOCVD (nine years), and LPE (ten years). He was the first to put VCSELs into volume production. He holds 37 U.S. patents, most of which are related to VCSELs. he has spent his entire postgraduate career working with the same organization where he started as a part of Honeywell in 1985, which became the VCSEL business unit and was purchased by Finisar, Allen, TX, in 2004. He is currently a Senior Engineering Fellow with the Advanced Optical Components Division of Finisar. $\mathrm{He}$ is currently developing 1310-nm, 8G 850-nm, and 17G 850-nm VCSELs.

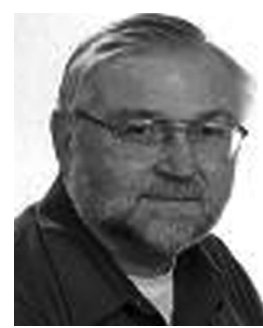

Gary A. Evans (S'69-M'75-SM' 82-F'92) was born in Omak, WA. He received the B.S.E.E. degree from the University of Washington, Seattle, in 1970 and the M.S.E.E. and Ph.D. degrees in electrical engineering and physics from the California Institute of Technology (Caltech), Pasadena, in 1971 and 1975, respectively.

After a postdoctoral year at Caltech, he was with R\&D Associates, Marina Del Rey, CA, and was a Visiting Assistant Professor with the Electrical Engineering Department, University of Washington (1977-1979). He has been with the Aerospace Corporation, El Segundo, CA (1979-1981), TRW (now Northrop Grumman), Redondo Beach, CA (1981-1984), and RCA Laboratories (now Sarnoff), Princeton, NJ (1984-1992). In 1992, he joined Southern Methodist University, Dallas, TX, as a Professor in the Electrical Engineering Department and is President of the SMU Faculty Senate. He is a founder and member of the board of directors of Photodigm and a member of the SMU Board of Trustees. Since 1979, he has primarily worked on the design, growth, and fabrication of conventional cleaved facet and grating surface-emitting semiconductor lasers. He has authored or coauthored over 260 publications, holds 35 patents, and is a coeditor of the book Surface Emitting Semiconductor Lasers (Academic, 1993). He is a Licensed Professional Engineer in Washington since 1979.

Dr. Evans has served on numerous national and international IEEE committees, is a past Chairman of the Princeton, NJ, and Dallas, TX, Sections of the Lasers and Electro-Optics Society (LEOS), a past Chairman of the Santa Monica Bay Section of the IEEE, was an Associate Editor of the IEEE JouRNAL OF QUANTUM ELECTRONICS (1989-1995), was the Finance Chairman for the 1994 IEEE International Semiconductor Laser Conference, and was a Technical Program Vice Chair for the 1996 International Communications Conference. 


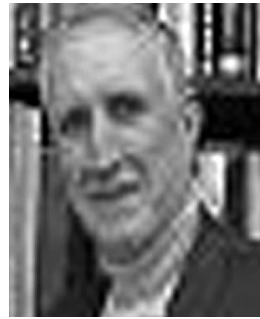

Jerome K. Butler (S'59-M'65-SM'78-F'89) was born in Shreveport, LA. He received the B.S.E.E. degree from Louisiana Polytechnic Institute, Ruston, and the M.S.E.E. and Ph.D. degrees from the University of Kansas, Lawrence.

He was a Research Assistant and held a CRES Fellowship at the Center for Research in Engineering Sciences, University of Kansas. He conducted research concerned with electromagnetic wave propagation and the optimization and synthesis techniques of antenna arrays. He joined the faculty of the School of Engineering, Southern Methodist University, Dallas, TX, where he is now a University Distinguished Professor of Electrical Engineering. His primary research areas are solid-state injection lasers, radiation and detection studies of lasers, millimeter-wave systems, integrated optics, and the application of integrated optical circuits, and quantum electronics. In summers from 1969 to 1990 , he was a Staff Scientist with the David Sarnoff Research Center (RCA Laboratories), Princeton, NJ. During the 1996-1997 academic year, he was on sabbatical leave with the Photonics and Micromachining System Components Laboratory, Texas Instruments. He has held consulting appointments with the Photonics and Micromachining System Components Laboratory at Texas Instruments, the Central Research Laboratory of Texas Instruments, Inc., the Geotechnical Corporation of Teledyne, Inc., Earl Cullum Associates, Dallas, TX, and the University of California Los Alamos Scientific Laboratory, Los Alamos, NM.

Dr. Butler is a member of Sigma Xi, Tau Beta Pi, and Eta Kappa Nu. He is a registered Professional Engineer in the State of Texas. In 1977, he was the recipient of the Southern Methodist University Sigma Xi Research Award. 